\title{
A One- and Two-Dimensional Least-Squares Smoothing and Edge-Sharpening Method for Image Processing
}

Mozelle R. Bell

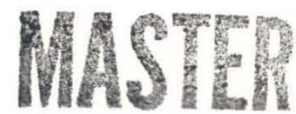

\section{OAK RIDGE NATIONAL LABORATORY}




\section{DISCLAIMER}

This report was prepared as an account of work sponsored by an agency of the United States Government. Neither the United States Government nor any agency Thereof, nor any of their employees, makes any warranty, express or implied, or assumes any legal liability or responsibility for the accuracy, completeness, or usefulness of any information, apparatus, product, or process disclosed, or represents that its use would not infringe privately owned rights. Reference herein to any specific commercial product, process, or service by trade name, trademark, manufacturer, or otherwise does not necessarily constitute or imply its endorsement, recommendation, or favoring by the United States Government or any agency thereof. The views and opinions of authors expressed herein do not necessarily state or reflect those of the United States Government or any agency thereof. 


\section{DISCLAIMER}

Portions of this document may be illegible in electronic image products. Images are produced from the best available original document. 
Printed in the United States of America. Available from

National Technical Information Service

U.S. Department of Commerce

5285 Port Royal Road, Springfield, Virginia 22161

Price: Printed Copy $\$ 5.00$; Microfiche $\$ 2.25$

This report was prepared as an account of work sponsored by the United States Government. Neither the United States nor the Energy Research and Development Administration, nor any of their employees, nor any of their contractors, subcontractors, or their employees, makes any warranty, express or implied, or assumes any legal liability or responsibility for the accuracy, completeness or usefulness of any information, apparatus, product or process disclosed, or represents that its use would not infringe privately owned rights. 
Contract No. W-7405-eng-26

Molecular Anatomy (MAN) Program

A ONE- AND TWO-DIMENSIONAL LEAST-SQUARES SMOOTHING AND EDGE-SHARPENING METHOD FOR IMAGE PROCESSING

Mozelle R. Bell 1,2

\section{JANUARY 1976}

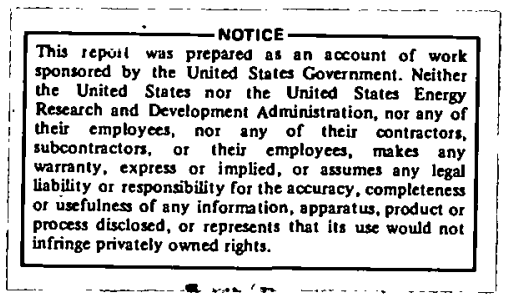

OAK RIDGE NATIONAL LABORATORY

Oak Ridge, Tennessee 37830 operated by

UNION CARBIDE CORPORATION

for the

U.S. ENERGY RESEARCH AND DEVELOPMENT ADMINISTRATION

$\bar{I}_{\text {Consultant }}$

${ }^{2}$ Communications about this report should be sent to: P. R. Bell, Medical Instrumentation Group, Building 9201-2, Post Office Box Y, Oak Ridge, Tennessee 37830 
THIS PAGE

WAS INTENTIONALLY

LEFT BLANK 
TABLE OF CONTENTS

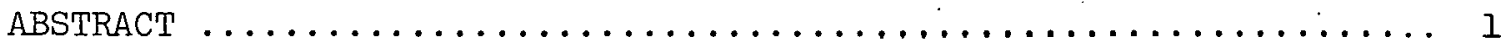

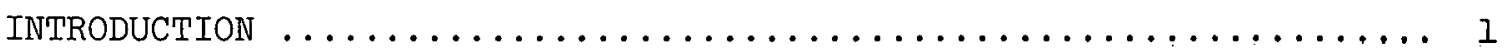

I. One Dimensional $\ldots \ldots \ldots \ldots \ldots \ldots \ldots \ldots \ldots \ldots \ldots \ldots \ldots, 3$

A. Orthogonal Polynomial Method .................... 3

1. Five Point Formulas for Polynomials of Degree $₫ 5 \ldots \ldots \ldots \ldots \ldots \ldots \ldots \ldots \ldots \ldots \ldots \ldots \ldots \ldots \ldots \ldots \ldots \ldots \ldots$

2. Seven Point Formulas for Polynomials of Degree $\leq 5 \ldots \ldots \ldots \ldots \ldots \ldots \ldots \ldots \ldots \ldots \ldots$

B. Least Squares Solutions Using a Symmetrical

Grid with Equal Spacing. ................... 7

1. Five Point Formulas ..................... 8

1.1. Quadratic or Cubic .................. 8

2. Seven Point Formulas .....................

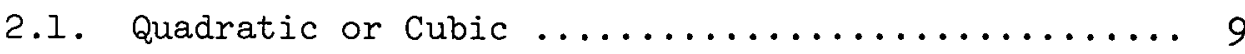

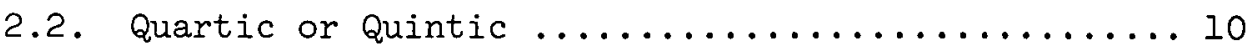

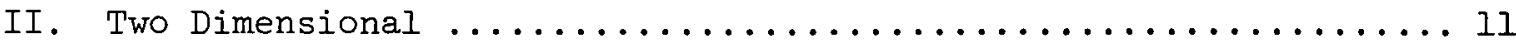

1. Determination of Central Value of Function and Second Derivative ....................... II

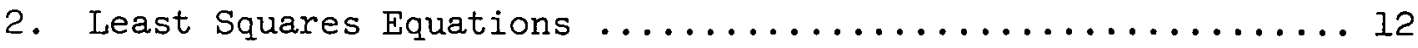

3. $3 \times 3$ Point Formulas ........................ 16

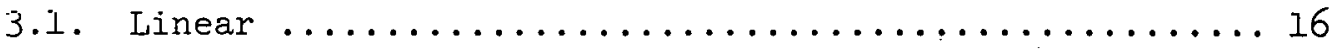

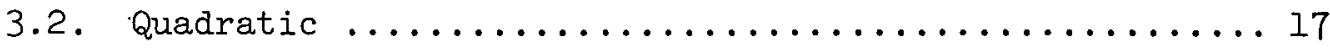

4. $5 \times 5$ Point Formulas ............................ 17

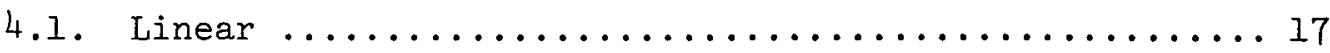

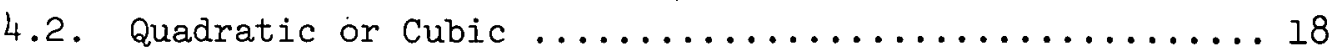

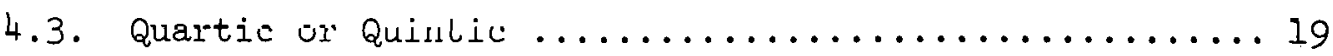

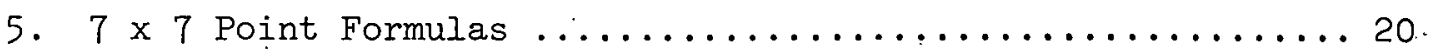

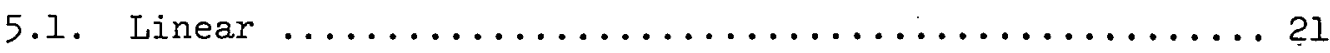

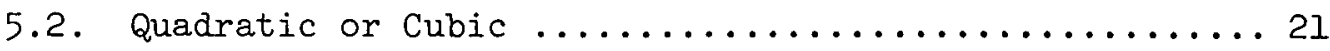

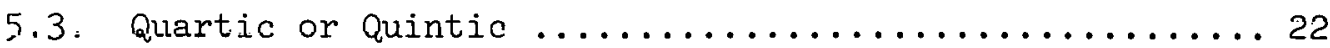




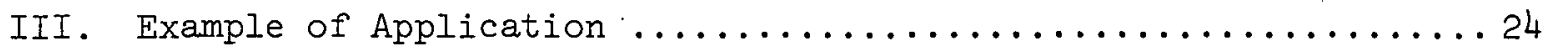

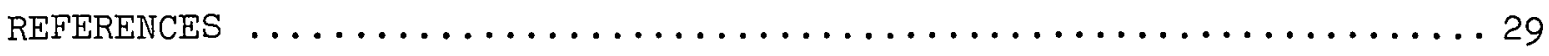

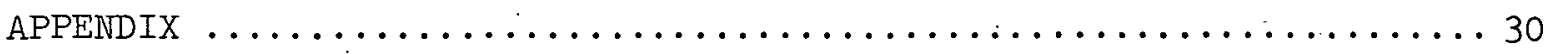


A ONE- AND TWO-DIMENSIONAL LEAST-SQUARES SMOOTHING AND EDGE-SHARPENING METHOD FOR IMAGE PROCESSING

Mozelle R. Bell

\section{ABSTRACT}

A rapid method is developed for two-dimensional smoothing and edge-sharpening by the least-squares fitting of a function to a limited area of the data. This convolution or matrix weighting is applied at each point of the data set to yield a smoothed or a sharpened image. Weighting matrices for $3 \times 3,5 \times 5$, and $7 \times 7$ point fitting areas are provided for polynomial function fits of all degrees up to the highest degree determinable. For the $7 \times 7$ point fitting area weights for fitting functions of up to the quartic in both dimensions are supplied. Application of the $5 \times 5$ point quadratic fit smoothing to a nuclear medicine image is shown as an example.

\section{INTRODUCTION}

Data smoothing is used extensively in nuclear medicine and other disciplines that employ images for analysis. Smoothing is needed to permit the use of isometric images, multicycle images, and color coded images, all of which suffer greatly from excessive raggedness of the data. Excess high frequency noise lying outside the frequency range defined by the system point source response reduces the readability of the image without contributing useful information. The smoothing methods generally employed in nuclear medicine are simple averages of neighboring points, of a $3 \times 3$ square area, or at best a simple Gaussian shaped weighting function. These methods are chosen for speed or simplicity of application and have little or no theoretical justification. The methods of least-squares two dimensional fitting give better results and have sound statistical justification but when applied in the usual straightforward manner are too time consuming for routine application to the large nuclear medicine images.

One-dimensional smoothing methods that provide least-squares fitted polynomials to noisy data are widely known. These yield good smoothing by a simple and rapid convolution or weighting method. Each entry in a short 
list of numbers derived from the Gram orthogonal polynomials ${ }^{1}$ or directly from least-squares methods ${ }^{2}$ is multiplied by successive data values. The sum of these products yields the least-squares fitted value at the midpoint of the range. The list of multipliers is then advanced along the data list by one point and the operation is repeated to provide a fitted value at the next position of the mid-point and so on. The method is rapid and well suited for use in small computers.

The method is here extended to two dimensions to provide a rapid twodimensional smoothing method.

Another procedure, of value especially to nuclear medicine where images have necessarily both poor resolution and bad statistical fluctuation, is that of boundary sharpening. The method of sharpening an edge where there should be a sudden transition, but where the transition has been broadened by inadequate resolution, is an old one ${ }^{3}$. It has frequently been applied to Beta ray or scintillation spectra. It consists in the subtraction of a certain amount of the second derivative of the data curve from the curve to yield sharper transitions. Extending this method to two dimensions requires the generation of the directionally averaged second derivative of the image at each data point and the subtraction of the resultant correction image from the original to produce a resultant image with sharpened organ boundaries.

The same methods that provide the least-squares fitted value at the central point of the weighting matrix area can be extended to provide similarly the directionally averaged second derivative of the fitted twodimensional function at the central point of the range. Weighting tables for $3 \times 3,5 \times 5$, and $7 \times 7$ point fitting regions are provided to yield smoothing and second derivative values for fitted functions of various degrees. The degree extends to a quartic in both dimensions for the $7 \times 7$ point area. The $7 \times 7$ point smoothing or second derivative procedures require about 3 minutes to process a 16,000 point nuclear medicine image on a PDP-8/E computer without EAE. 


\section{One Dimensional}

A. Orthogonal Polynomial Method

The use of the Gram orthogonal polynomials to calculate the leastsquare formulas for $\mathrm{n}^{\text {th }}$ degree polynomials using $2 \mathrm{M}+1$ points has been expounded in great detail in F. B. Hildebrand's "Introduction to Numerical Analysis"l in sections 7.11 through 7.14 (see pages 288 through. 302). [Formula numbers with $H$ given in the remainder of this memo refer to this book]. Pormulas are given there for the smoothed central value for cases up to $\mathrm{n}=5$ and $\mathrm{M}=3$ (i.e., fifth-degree seven-point formulas). For the sake of completeness we develop and repeat these formulas here and also include the companion formulas for the second derivative at the central point, since these will later be found to be useful for edge-sharpening of the data.

1. Five Point Formulas for Polynomials of Degree $\leq 3$. For $M=2$ ( 5 points) the highest order orthogonal polynomial needed is of third degree, $p_{3}(t, 4)$; a 4 th degree polynomial would fit the data exactly. Here $t$ represents the distance from the mid-point of the range in units of the spacing.

Then $y(t)=a_{0} p_{0}(t, 4)+a_{1} p_{1}(t, 4)+a_{2} p_{2}(t, 4)+a_{3} p_{3}(t, 4)$. IA.I.I

Substituting the values for the orthogonal polynomials given by formulas $7.2 .1 \mathrm{H}$ we have

$$
y(t)=a_{0}+a_{1} \frac{t}{2}+\frac{a_{2}}{2}\left(t^{2}-2\right)+\frac{a_{3}}{6}\left(5 t^{3}-17 t\right)
$$

whence

$$
y^{\prime \prime}(t)=a_{2}+5 a_{3} t
$$

For the central point, $t=0$,

$$
y(0)=a_{0}-a_{2}
$$

and

$$
y^{\prime \prime}(0)=a_{2}
$$


We note that at the central point the cubic term in the original equation makes no contribution. The quadratic and cubic fits give the same results at the central point.

Applying equations $7.11 .14 \mathrm{H}$ and $7.11 .13 \mathrm{H}$ and noting that $p_{0}(t, 4)=1$ and $p_{2}(t, 4)=\frac{\left(t^{2}-2\right)}{2}$ we have

$$
\begin{aligned}
& \gamma_{0}=\sum_{t=-2}^{t=2} p_{0}^{2}(t, 4)=1^{2}+1^{2}+1^{2}+1^{2}+1^{2}=5 \\
& \gamma_{2}=\sum_{t=-2}^{t=2} p_{2}^{2}(t, 4)=\left(\frac{1}{2}\right)^{2}\left[(2)^{2}+(-1)^{2}+(-2)^{2}+(-1)^{2}+(2)^{2}\right]=\frac{7}{2} \\
& a_{0}=\frac{1}{\gamma_{0}} \sum_{t=-2}^{t=2} f(t) p_{0}(t, 4)=\frac{1}{5}\left[f_{-2}+f_{-1}+f_{0}+f_{1}+f_{2}\right] \\
& a_{2}=\frac{1}{\gamma_{2}} \sum_{t=-2} f(t) p_{2}(t, 4)=\frac{2}{7}\left[f_{-2}-1 / 2 f_{-1}-f_{0}-1 / 2 f_{1}+f_{2}\right]
\end{aligned}
$$

Here $f_{-2}, f_{-1}, \ldots, f_{2}$ represent the data values at $t=-2,-1, \ldots, 2$. Hence for a polynomial fit of either degree 2 or degree 3

$$
y(0)=a_{0}-a_{2}=\frac{1}{35}\left[-3 f_{-2}+12 f_{-1}+17 f_{0}+12 f_{1}-3 f_{2}\right]
$$

and

$$
y^{\prime \prime}(0)=a_{2}=\frac{2}{7}\left[f_{-2}-1 / 2 f_{-1}-f_{0}-1 / 2 f_{1}+f_{2}\right]
$$

2. Seven Point Formulas for Polynomials of Degree $\leq 5$. For $M=3$ ( 7 points) the highest order least squares fit possible is

$$
\begin{aligned}
y(t) & =a_{0} p_{0}(t, 6)+a_{1} p_{1}(t, 6)+a_{2} p_{2}(t, 6)+a_{3} p_{3}(t, 6) \\
& +a_{4} p_{4}(t, 6)+a_{5} p_{5}(t, 6)
\end{aligned}
$$


Evaluating formulas $7.11 .10 \mathrm{H}$ with $\mathrm{M}=3$ to obtain the orthogonal polynomials for this case and substituting into (2.1),

$$
\begin{aligned}
y(t) & =a_{0}+a_{1} \frac{t}{3}+a_{2}\left(\frac{t^{2}-4}{5}\right)+a_{3}\left(\frac{t^{3}-7 t}{6}\right)+a_{4}\left(\frac{7 t^{4}-67 t^{2}+72}{36}\right) \\
& +a_{5}\left(\frac{21 t^{5}-245 t^{3}+524 t}{60}\right)
\end{aligned}
$$

whence

$$
y^{\prime \prime}(t)=\frac{2 a_{2}}{5}+a_{3} t+\frac{7}{3} a_{4} t^{2}-\frac{67}{18} a_{4}+7 a_{5} t^{3}-\frac{49}{2} a_{5} t
$$

For the central point, $t=0$,

$$
y(0)=a_{0}-\frac{4 a_{2}}{5}+2 a_{4}
$$

and

$$
\mathrm{y}^{\prime \prime}(0)=\frac{2 \mathrm{a}_{2}}{5}-\frac{67}{18} \mathrm{a}_{4}
$$

Again applying equations $7.11 .14 \mathrm{H}$ and $7.11 .13 \mathrm{H}$ we have

$$
\begin{aligned}
& \gamma_{0}=\sum_{t=-3}^{t=+3}(1)^{2}=7 \\
& \gamma_{2}=\sum_{t=-3}^{t=+3}\left[\frac{t^{2}-4}{5}\right]^{2}=1+0+\frac{9}{25}+\frac{16}{25}+\frac{9}{25}+0+1=\frac{84}{25} \\
& \gamma_{4}=\sum_{t=-3}^{t-+3}\left[\frac{7 t^{4}-67 t^{2}+72}{36}\right]^{2}=1+\frac{49}{9}+\frac{1}{9}+4+\frac{1}{9}+\frac{49}{9}+1=\frac{154}{9} \\
& a_{0}=\frac{1}{\gamma_{0}} \sum_{t=-3}^{t=+3} f(t)(1)=\frac{1}{7}\left[f_{-3}+f_{-2}+f_{-1}+f_{0}+f_{1}+f_{2}+f_{3}\right] .
\end{aligned}
$$

$\perp A . \dot{2} \cdot \dot{9}$ 


$$
\begin{aligned}
a_{2}= & \frac{1}{\gamma_{2}} \sum_{t=-3}^{t=+3} f(t)\left[\frac{t^{2}-4}{5}\right]=\frac{25}{84}\left[f_{-3}+0-\frac{3}{5} f_{-1}-\frac{4}{5} f_{0}\right. \\
& \left.-\frac{3}{5} f_{1}+0+f_{3}\right] \\
a_{4}= & \frac{1}{\gamma_{4}} \sum_{t=-3}^{t=+3} f(t)\left[\frac{7 t^{4}-67 t^{2}+72}{36}\right]=\frac{9}{154}\left[f_{-3}-\frac{7}{3} f_{-2}\right. \\
& \left.+\frac{1}{3} f_{-1}+2 f_{0}+\frac{1}{3} f_{1}-\frac{7}{3} f_{2}+f_{3}\right]
\end{aligned}
$$

For a polynomial fit of degree 2 or degree $3, a_{4}$ and $a_{5}$ do not occur and

$$
\begin{aligned}
y(0) & =a_{0}-\frac{4}{5} a_{2}=\frac{1}{21}\left[-2 f_{-3}+3 f_{-2}+6 f_{-1}+7 f_{0}+6 f_{1}\right. \\
& \left.+3 f_{2}-2 f_{3}\right]
\end{aligned}
$$

and

$$
y^{\prime \prime}(0)=\frac{2 a_{2}}{5}=\frac{5}{42}\left[f_{-3}-\frac{3}{5} f_{-1}-\frac{4}{5} f_{0}-\frac{3}{5} f_{1}+f_{3}\right]
$$

For a polynomial fit of degree 4 or degree 5 ,

$$
\begin{aligned}
y(0) & =a_{0}-\frac{4}{5} a_{2}+2 a_{4}=\frac{1}{231}\left[5 f_{-3}-30 f_{-2}+75 f_{-1}+131 f_{0}\right. \\
& \left.+75 f_{1}-30 f_{2}+5 f_{-3}\right] .
\end{aligned}
$$

and

$$
\begin{aligned}
y^{\prime \prime}(0) & =\frac{2 a_{2}}{5}-\frac{67}{18} a_{4}=\frac{1}{132}\left[-13 f_{-3}+67 f_{-2}-19 f_{-1}-70 f_{0}\right. \\
& \left.-19 f_{1}+67 f_{2}-13 f_{3}\right]
\end{aligned}
$$


B. Least Squares Solutions Using a Symmetrical Grid with Equal Spacing

Let us determine our experimental data points in the following symmetrical types of grid:

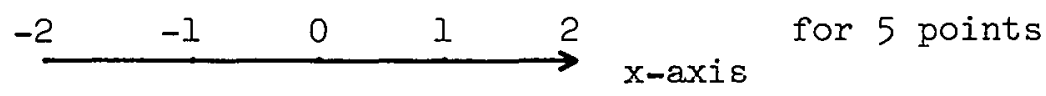

a.nd

$\begin{array}{lllllll}-3 & -2 & -1 & 0 & 1 & 2 & 3 \\ \longrightarrow\end{array}$

We then have $\sum_{i=1}^{n} x_{i}=\sum_{i=1}^{n} x_{i}^{3}=\sum_{i=1}^{n} x_{i}^{5}=\ldots=\sum_{i=1}^{n} x_{i}^{\text {odd }}=0$.

As we shall now show this fact allows us to make such great simplifications in the set of least-square equations that the solutions thereof may be obtained directly. This provides an alternate method of solution in which the use and knowledge of orthogonal polynomials is not needed.

Let us represent a general fitting polynomial of degree $\leq 5$ by

$$
y=b_{1}+b_{2} x+b_{3} x^{2}+b_{4} x^{3}+b_{5} x^{4}+b_{6} x^{5}
$$

Then $y(0)=b_{1}$

and $y^{\prime \prime}(0)=2 b_{3}$

We can reduce this from a quintic to a quartic to a culyis to a quadratic by setting $b_{6}=0, b_{5}=0$, and $b_{4}=0$ respectively.

The least-square equations are obtained by minimizing

$$
F \equiv \sum_{i=1}^{n}\left[f_{i}-\left(b_{1}+b_{2} x_{i}+b_{3} x_{i}^{2}+b_{4} x_{i}^{3}+b_{5} x_{i}^{4}+b_{6} x_{i}^{5}\right)\right]^{2}
$$

where $f_{i}$ is the experimental data.

Differentiating $F$ with respect to $b_{1}, b_{2}, b_{3}, b_{4}, b_{5}$, and $b_{6}$ in turn, setting each of the six equations equal to 0 and remembering that $\Sigma x_{i}$ odd $=0$, we get the following set of least-squares equations. 


$$
\begin{aligned}
& \frac{\partial F}{\partial b_{1}}: \quad c_{1} \equiv \Sigma f_{i}=n b_{1}+b_{3} \sum x_{i}^{2}+b_{5} \sum x_{i}^{4} \\
& \frac{\partial F}{\partial b_{2}}: \quad c_{2} \equiv \Sigma f_{i} x_{i}=b_{2} \sum x_{i}^{2}+b_{4} \sum x_{i}^{4}+b_{6} \sum x_{i}^{6} \\
& \frac{\partial F}{\partial b_{3}}: \quad c_{3} \equiv \Sigma f_{i} x_{i}{ }^{2}=b_{1} \sum x_{i}{ }^{2}+b_{3} \sum x_{i}{ }^{4}+b_{5} \sum x_{i}{ }^{6} \\
& \frac{\partial F}{\partial b_{4}}: \quad c_{4} \equiv \sum f_{i} x_{i}^{3}=b_{2} \sum x_{i}^{4}+b_{4} \sum x_{i}^{6}+b_{6} \sum x_{i}^{8} \\
& \frac{\partial F}{\partial b_{5}}: \quad c_{5} \equiv \Sigma f_{i} x_{i}^{4}=b_{1} \sum x_{i}^{4}+b_{3} \sum x_{i}^{6}+b_{5} \sum x_{i}^{8} \\
& \frac{\partial F}{\partial b_{6}}: \quad c_{6} \equiv \Sigma f_{i} x_{i}^{5}=b_{2} \sum x_{i}^{6}+b_{4} \sum x_{i}^{8}+b_{6} \sum x_{i}^{10}
\end{aligned}
$$

For determining central values only the three equations B.5, B.7, and B.9 need be considered.

The solutions of the least-squares equations will be given as linear functions of $\mathrm{C}_{1}, \mathrm{C}_{3}$, and $\mathrm{C}_{5}$, which are themselves linear functions of $\mathrm{f}_{i}$. We will use these facts to find a set of weights, $w_{i}$, such that our answers may be written as $\sum_{i=1}^{n} w_{i} f_{i}$, an easily calculable form.

1. Five Point Formulas.

$$
\begin{aligned}
& \text { For } \mathrm{n}=5: \mathrm{x}_{1}=-2, \mathrm{x}_{2}=-1, \mathrm{x}_{3}=0, \mathrm{x}_{4}=1, \mathrm{x}_{5}=2 \text {, whence } \\
& \sum \Sigma \mathrm{x}_{i}^{2}=10 ; \sum \mathrm{x}_{i}^{4}=34 ; \Sigma \mathrm{x}_{i}^{6}=130 ; \sum \mathrm{x}_{i}{ }^{8}=514 .
\end{aligned}
$$

1.1. Quadratic or Cubic (highest possible degree for $n=5$ ). For a quadratic or cubic $\left(b_{5}=b_{6} \equiv 0\right)$ the set of equations to be solved becomes

$$
\begin{aligned}
& c_{1}=5 b_{1}+10 b_{3} \\
& c_{3}=10 b_{1}+34 b_{3}
\end{aligned}
$$


The solution of this pair of equations gives

$$
\begin{aligned}
& y(0)=b_{1}=\frac{1}{35}\left(17 c_{1}-5 c_{3}\right) \\
& y^{\prime \prime}(0)=2 b_{3}=\frac{1}{7}\left(c_{3}-2 c_{1}\right)
\end{aligned}
$$

Apn]ying the definitions of $\mathrm{C}_{1}$ and $\mathrm{C}_{3}$

$$
y(0)=\frac{1}{35}\left[-3 f_{-2}+12 f_{-1}+17 f_{0}+12 f_{1}-3 f_{2}\right]
$$

and

$$
y^{\prime \prime}(0)=\frac{1}{7}\left[2 f_{-2}-f_{-1}-2 f_{0}-f_{1}+2 f_{2}\right]
$$

in agreement with equations IA.I.10 and IA.I.Il, where $f$ with a subscript means the value of the data at the $x$ point which is the subscript.

2. Seven Point Formulas.

$$
\begin{aligned}
& \text { For } \mathrm{n}=7: \mathrm{x}_{1}=-3 ; \mathrm{x}_{2}=-2 ; \mathrm{x}_{3}=-1 ; \mathrm{x}_{4}=0 ; \mathrm{x}_{5}=1 ; \mathrm{x}_{6}=2 ; \\
& \mathrm{x}_{7}=3 \text { whence } \\
& \quad \sum \mathrm{x}_{i}^{2}=28 ; \sum \mathrm{x}_{i}^{4}=196 ; \sum \mathrm{x}_{i}^{6}=1588 ; \sum \mathrm{x}_{i}^{8}=13636 .
\end{aligned}
$$

2.1. Quadratic or Cubic. For a quadratic or cubic the set of equations to be solved is:

$$
\begin{aligned}
& c_{1}=7 b_{1}+28 b_{3} \\
& c_{3}=28 b_{1}+196 b_{3}
\end{aligned}
$$

The solution of this pair of equations gives

$$
\begin{aligned}
& \mathrm{y}(0)=\mathrm{b}_{1}=\frac{1}{21}\left(7 \mathrm{C}_{1}-\mathrm{C}_{3}\right) \\
& \mathrm{y}^{\prime \prime}(0)=2 \mathrm{~b}_{3}=\frac{1}{42}\left(\mathrm{c}_{3}-4 \mathrm{c}_{1}\right)
\end{aligned}
$$


Expressed in terms of the experimental data these are

$$
y(0)=\frac{1}{21}\left(-2 f_{-3}+3 f_{-2}+6 f_{-1}+7 f_{0}+6 f_{1}+3 f_{2}-2 f_{3}\right) \quad \text { IB. } 2 \cdot 1 \cdot 5
$$

a nd

$$
y^{\prime \prime}(0)=\frac{1}{42}\left(5 f_{-3}+0-3 f_{-1}-4 f_{0}-3 f_{1}+0+5 f_{3}\right) \quad \text { IB.2.1.6 }
$$

in agreement with equations IA.2.12 and IA.2.13.

2.2. Quartic or Quintic (highest possible degree for $n=7$ ). For a quartic or quintic the set of equations to be solved is

$$
\begin{aligned}
& c_{1}=7 b_{1}+28 b_{3}+196 b_{5} \\
& c_{3}=28 b_{1}+196 b_{3}+1588 b_{5} \\
& c_{5}=196 b_{1}+1588 b_{3}+13636 b_{5}
\end{aligned}
$$

Although the arithmetic involved is more tedious than in previous cases, the solution is straightforward and gives

$$
y(0)=b_{1}=\frac{524 C_{1}-245 C_{3}+21 c_{5}}{924}
$$

and

$$
y^{\prime \prime}(0)=2 b_{3}=\frac{-840 c_{1}+679 c_{3}-67 c_{5}}{1584}
$$

Expressed in terms of the experimental data these are

$$
\begin{aligned}
y(0) & =\frac{1}{231}\left(5 f_{-3}-30 f_{-2}+75 f_{-1}+13 f_{0}+75 f_{1}-30 f_{2}\right. \\
& \left.+5 f_{3}\right)
\end{aligned}
$$

and

$$
\begin{aligned}
y^{\prime \prime}(0) & =\frac{1}{132}\left(-13 f_{-3}+67 f_{-2}-19 f_{-1}-70 f_{0}-19 f_{1}+67 f_{2}\right. \\
& \left.-13 f_{3}\right)
\end{aligned}
$$

in agreement with equations IA.2.14 and IA.2.15. 


\section{Two Dimensional}

1. Determination of Central Value of Function and Second Derivative.

We shall here generalize the symmetric grid procedure described in Section I, Part B.

Let us represent a general fitting polynomial of degree $\leq 5$ in two dimensions by

$$
\begin{aligned}
f(x, y) & =b_{1}+b_{2} x+b_{3} y+b_{4} x^{2}+b_{5} x^{2} y^{2}+b_{6} y^{2}+b_{7} x^{3} \\
& +b_{8} x^{2} y+b_{9} x y^{2}+b_{10} y^{3}+b_{11} x^{4}+b_{12} x^{3} y+b_{13} x^{2} y^{2} \\
& +b_{14} x y^{3}+b_{15} y^{4}+b_{16^{2}}+b_{17} x^{4} y+b_{18} x^{3} y^{2}+b_{19} x^{2} y^{3} \\
& +b_{20} x y^{4}+b_{21} y^{5}
\end{aligned}
$$

Then

$$
f(0,0)=b_{1}
$$

We can reduce II.I.I from a quintic to a quartic by setting $b_{16}$ through $\mathrm{b}_{21} \equiv 0$; to a cubic by additionally setting $\mathrm{b}_{11}$ through $\mathrm{b}_{15} \equiv 0$; to a quadratic by additionally setting $b_{7}$ through $b_{10} \equiv 0$.

The directional derivative $\frac{d f}{d s}$ at any point $(x, y)$ taken in the direction of a straight line making an angle $\alpha$ with the $x$-axis is

$\frac{d f(x, y)}{d s}=\frac{\partial f}{\partial x} \cos \alpha+\frac{\partial f}{\partial y} \sin \alpha \equiv f_{x} \cos \alpha+f_{y} \sin \alpha$

The second directional derivative is

$$
\frac{d^{2} f}{d s^{2}}(x, y)=\frac{\partial\left[f_{x} \cos \alpha+f_{y} \sin \alpha\right]}{\partial x} \cos \alpha+\frac{\partial\left[f_{x} \cos \alpha+f_{y} \sin \alpha\right]}{\partial y} \operatorname{II.I.4}
$$

or

$$
\frac{d^{2} f}{d s^{2}}(x, y)=f_{x x} \cos ^{2} \alpha+2 f_{y x} \sin \alpha \cos \alpha+f_{y y} \sin ^{2} \alpha
$$


since $f_{y x}=f_{x y}$. We take the average of $f^{\prime \prime}(x, y)$ over $\alpha$ by noting that $\left(\cos ^{2} \alpha\right)_{a v}=\left(\sin ^{2} \alpha\right)_{a v}=\frac{1}{2 \pi}{ }_{0}^{2 \pi} \cos ^{2} \alpha d \alpha=\frac{1}{2 \pi} \sin ^{2} \alpha=\frac{1}{2}$, and $(\sin \alpha \cos \alpha)_{a v}$ $=\frac{1}{2 \pi}{ }_{0}^{2 \pi} \sin \alpha \cos \alpha d \alpha=0$. Thus

$$
f^{\prime \prime}(x, y)_{a v}=\frac{1}{2}\left(f_{x x}+f_{y y}\right)
$$

From equation II.I.I

$$
f^{\prime \prime}(0,0)_{a v}=b_{4}+b_{6} .
$$

When comparing one-dimensional answers as special cases of the twodimensional results, note that since the two-dimensional result is an average it gives only $1 / 2$ the one-dimensional results in the $\mathrm{x}$ and $\mathrm{y}$ directions, respectively.

2. Least-Squares Equations. The least-square equations are obtained by minimizing

$$
\begin{aligned}
& \tilde{F} \equiv \sum_{i=1}^{n} \sum_{j=1}^{n}\left[f_{i j}-\left(b_{1}+b_{2} x+b_{3} y+b_{4} x^{2}+b_{5} x y+b_{6} y^{2}\right.\right. \\
& \quad+b_{7} x^{3}+b_{8} x^{2} y+b_{9} x y^{2}+b_{10} y^{3}+b_{11} x^{4}+b_{12} x^{3} y+b_{13} x^{2} y^{2} \\
&+b_{14} x y^{3}+b_{15} y^{4}+b_{16^{2}} x^{5}+b_{17} x^{4} y+b_{18} x^{3} y^{2}+b_{19} x^{2} y^{3} \\
&\left.+b_{20} x y^{4}+b_{21} y^{5}\right]^{2}
\end{aligned}
$$

where $f_{i j}$ is the experimental data. Differentiating $F$ with respect to $b_{1}, b_{2}, b_{3}, \ldots, b_{21}$ in turn, setting each of the 21 equations equal to 0 and remembering that $\Sigma x_{i}{ }^{\text {odd }}=\Sigma y_{i}$ odd $=0$, we get the following set of least-square equations. 


$$
\begin{aligned}
\frac{\partial F}{\partial b_{1}}: & k_{1} \equiv \sum_{i} \sum_{j} f_{i j}=n^{2} b_{1}+n \Sigma x_{i}{ }^{2} b_{4}+n \Sigma y_{j}{ }^{2} b_{6}+n \sum x_{i}{ }^{4} b_{11}+\sum x_{i}{ }^{2} \sum y_{j}{ }^{2} b_{13} \\
& +n \Sigma y_{j}{ }^{4} b_{15}
\end{aligned}
$$

$$
\begin{aligned}
\frac{\partial F}{\partial b_{2}}: & k_{2} \equiv \sum_{i} \sum_{j} f_{i j} x_{i}=n \Sigma x_{i}^{2} b_{2}+n \Sigma x_{i}^{4} b_{7}+\Sigma x_{i}{ }^{2} \Sigma y_{j}{ }^{2} b_{9}+n \Sigma x_{i} b_{16} \\
& +\Sigma x_{i}^{4} \Sigma y_{j} b_{18}+\Sigma x_{i}^{2} \Sigma y_{j}{ }^{4} b_{20}
\end{aligned}
$$

$$
\begin{aligned}
\frac{\partial F}{\partial b_{3}}: & k_{3} \equiv \sum_{i} \sum_{j} f_{i j} y_{j}=n \Sigma y_{j}{ }^{2} b_{3}+\Sigma x_{i}{ }^{2} \Sigma y_{j}{ }^{2} b_{8}+n \Sigma y_{j}{ }^{4} b_{10}+\Sigma x_{i}{ }^{4} \Sigma y_{j}{ }^{2} b_{17} \\
& +\Sigma x_{i}{ }^{2} \sum y_{j}{ }^{4} b_{19}+n \Sigma y_{j}{ }^{4} b_{21}
\end{aligned}
$$

$$
\begin{aligned}
\frac{\partial F}{\partial b_{4}}: & k_{4} \equiv \sum_{i} \sum_{j} f_{i j} x_{i}^{2}=n \Sigma x_{i}^{2} b_{1}+n \Sigma x_{i}{ }^{4} b_{4}+\sum x_{i}{ }^{2} \sum y_{j}{ }^{2} b_{6}+n \Sigma x_{i}{ }^{6} b_{11} \\
& +\sum x_{i}{ }^{4} \sum y_{j}{ }^{2} b_{13}+\sum x_{i}{ }^{2} \Sigma y_{j}{ }^{4} b_{15}
\end{aligned}
$$

$$
\frac{\partial F}{\partial b_{5}}: \quad k_{5} \equiv \sum_{i} \sum_{j} f_{i j} x_{i} y_{j}=\Sigma x_{i}{ }^{2} \Sigma y_{j}{ }^{2} b_{5}+\Sigma x_{i}{ }^{4} \Sigma y_{j}{ }^{2} b_{12}+\Sigma x_{i}{ }^{2} \Sigma y_{j}{ }^{4} b_{14}
$$

$$
\begin{aligned}
\frac{\partial F}{\partial b_{6}}: & k_{6} \equiv \sum_{i} \sum_{j} f_{i j} y_{j}{ }^{2}=n \Sigma y_{j}{ }^{2} b_{1}+\Sigma x_{i}{ }^{2} \Sigma y_{j}{ }^{2} b_{4}+n \Sigma y_{j}{ }^{4} b_{6}+\Sigma x_{i}{ }^{4} \Sigma y_{j}{ }^{2} b_{11} \\
& +\Sigma x_{i}{ }^{2} \Sigma y_{j}{ }^{4} b_{13}+n \Sigma y_{j}{ }^{6} b_{15}
\end{aligned}
$$

$$
\begin{aligned}
\frac{\partial F}{\partial b_{7}}: & k_{7} \equiv \underset{i}{\sum} \sum_{j} \rho_{i j} x_{i}^{3}=n \Sigma x_{i}{ }^{4} b_{2}+n \Sigma x_{i}{ }^{6} b_{7}+\Sigma x_{i}{ }^{4} \Sigma y_{j}{ }^{2} b_{9}+n \Sigma x_{i}{ }^{8} b_{16} \\
& +\Sigma x_{i}{ }^{6} \sum y_{j}{ }^{2} b_{18}+\Sigma x_{i}{ }^{4} \Sigma y_{j}{ }^{4} b_{20}
\end{aligned}
$$




$$
\begin{aligned}
\frac{\partial F}{\partial b_{8}}: & k_{8} \equiv \sum_{i} \sum_{j} f_{i j} x_{i}{ }^{2} y_{j}=\sum x_{i}{ }^{2} \sum y_{j}{ }^{2} b_{3}+\sum x_{i}{ }^{4} \Sigma y_{j}{ }^{2} b_{8}+\sum x_{i}{ }^{2} \sum y_{j}{ }^{4} b_{10} \\
& +\sum x_{i}{ }^{6} \sum y_{j}{ }^{2} b_{17}+\sum x_{i}{ }^{4} \sum y_{j}{ }^{4} b_{19}+\sum x_{i}{ }^{2} \Sigma y_{j}{ }^{6} b_{2 l}
\end{aligned}
$$

$$
\begin{aligned}
& \frac{\partial F}{\partial b_{9}}: \quad k_{9} \equiv \sum_{i} \sum_{j} \cdot f_{i j} x_{i} y_{j}^{2}=\sum x_{i}{ }^{2} \Sigma y_{j}{ }^{2} b_{2}+\sum x_{i}{ }^{4} \Sigma y_{j}{ }^{2} b_{7}+\sum x_{i}{ }^{2} \Sigma y_{j}{ }^{4} b_{9} \\
& +\Sigma x_{i}{ }^{6} \Sigma y_{j}{ }^{2} b_{16}+\Sigma x_{i}{ }^{4} \Sigma y_{j}{ }^{4} b_{18}+\sum x_{i}{ }^{2} \Sigma y_{j}{ }^{6} b_{20}
\end{aligned}
$$

$$
\begin{gathered}
\frac{\partial F}{\partial b_{10}}: k_{10} \equiv \sum_{i} \sum_{j} f_{i j} y_{j}^{3}=n \Sigma y_{j}{ }^{4} b_{3}+\sum x_{i}{ }^{2} \sum y_{j}{ }^{4} b_{8}+n \Sigma y_{j} b_{10} \\
+\sum x_{i}{ }^{4} \Sigma y_{j}{ }^{4} b_{17}+\sum x_{i}{ }^{2} \Sigma y_{j}{ }^{6} b_{19}+n \Sigma y_{j}{ }^{8} b_{21}
\end{gathered}
$$

$$
\begin{gathered}
\frac{\partial F}{\partial b_{11}}: \quad k_{11} \equiv \sum_{i} \sum_{j} f_{i j} x_{i}{ }^{4}=n \sum x_{i}{ }^{4} b_{1}+n \sum x_{i}{ }^{6} b_{4}+\sum x_{i}{ }^{4} \sum y_{j}{ }^{6} b_{6} \\
+n \sum x_{i}{ }^{8} b_{11}+\sum x_{i}{ }^{6} \sum y_{j}{ }^{2} b_{13}+\sum x_{i}{ }^{4} \sum y_{j}{ }^{4} b_{15}
\end{gathered}
$$

$$
\begin{aligned}
\frac{\partial F}{\partial b_{12}}: k_{12} & \equiv \sum_{i} \sum_{j} f_{i j} x_{i}{ }^{3} y_{j}=\sum x_{i}{ }^{4} \sum y_{j}{ }^{2} b_{5}+\sum x_{i}{ }^{6} \sum y_{j}{ }^{2} b_{12} \\
& +\sum x_{i}{ }^{4} \sum y_{j}{ }^{4} b_{14}
\end{aligned}
$$

$$
\begin{aligned}
\frac{\partial F}{\partial b_{13}}: & k_{13} \equiv \sum_{i} \sum_{j} f_{i j} x_{i}{ }^{2} y_{j}{ }^{2}=\sum x_{i}{ }^{2} \Sigma y_{j}{ }^{2} b_{1}+\sum x_{i}{ }^{4} \Sigma y_{j}{ }^{2} b_{4}+\sum x_{i}{ }^{2} \Sigma y_{j}{ }^{4} b_{6} \\
& +\sum x_{i}{ }^{6} \sum y_{j}{ }^{2} b_{11}+\sum x_{i}{ }^{4} \sum y_{j}{ }^{4} b_{i 3}+\sum x_{i}{ }^{2} \sum y_{j}{ }^{6} b_{15}
\end{aligned}
$$

$$
\begin{aligned}
\frac{\partial F}{\partial b_{14}}: & k_{14} \equiv \sum_{i} \sum_{j} f_{i j} x_{i} y_{j}^{3}=\sum x_{i}{ }^{2} \sum y_{j}{ }^{4} b_{5}+\sum x_{i}{ }^{4} \sum y_{j}{ }^{4} b_{12} \\
& +\sum x_{i}{ }^{2} \sum y_{j}{ }^{6} b_{14}
\end{aligned}
$$




$$
\begin{aligned}
& \frac{\partial F}{\partial b_{15}}: \quad k_{15} \equiv \sum_{i} \sum_{j} f_{i j} y_{j}^{4}=n \sum y_{j}{ }^{4} b_{1}+\sum x_{i}{ }^{2} \sum y_{j} b_{4}+n \Sigma y_{j} b_{6}+\sum x_{i}{ }^{4} \sum y_{j}{ }^{4} b_{11} \\
& +\Sigma x_{i}^{2} \sum y_{j} b_{13}+n \Sigma y_{j}^{8} b_{15} \\
& \frac{\partial F}{\partial b_{16}}: \quad k_{16} \equiv \sum_{i} \sum_{j} f_{i j} x_{i}^{5}=n \sum x_{i}{ }^{6} b_{2}+n \sum x_{i}{ }^{8} b_{7}+\sum x_{i}{ }^{6} y_{j}{ }^{2} b_{9}+n \sum x_{i}{ }^{10} b_{16} \\
& +\sum x_{i}^{8} \sum y_{j}{ }^{2} b_{18}+\sum x_{i}{ }^{6} y_{j}{ }^{4} b_{20} \\
& \frac{\partial F}{\partial b_{17}}: \quad k_{17} \equiv \sum_{i} \sum_{j} f_{i j} x_{i}{ }^{4} y_{j}=\sum x_{i}{ }^{4} \Sigma y_{j}{ }^{2} b_{3}+\sum x_{i}{ }^{6} \sum y_{j}{ }^{2} b_{8}+\sum x_{i}{ }^{4} \sum y_{j}{ }^{4} b_{10} \\
& +\sum x_{i}{ }^{4} \sum y_{j}{ }^{6} b_{2 l}+\sum x_{i}{ }^{8} \sum y_{j}{ }^{2} b_{17}+\sum x_{i}{ }^{6} \sum y_{j}{ }^{4} b_{19}
\end{aligned}
$$

$$
\begin{aligned}
\frac{\partial F}{\partial b_{18}}: & k_{18} \equiv \sum_{i} \sum_{j} f_{i j} x_{i}{ }^{3} y_{j}{ }^{2}=\Sigma x_{i}{ }^{4} \Sigma y_{j}{ }^{2} b_{2}+\sum x_{i}{ }^{6} \Sigma y_{j}{ }^{2} b_{7}+\sum x_{i}{ }^{4} \Sigma y_{j}{ }^{4} b_{9} \\
& +\sum x_{i}{ }^{8} \sum y_{j}{ }^{2} b_{16}+\sum x_{i}{ }^{6} \Sigma y_{j}{ }^{4} b_{18}+\Sigma x_{i}{ }^{4} \Sigma y_{j}{ }^{6} b_{20}
\end{aligned}
$$

$$
\begin{aligned}
& \frac{\partial F}{\partial b_{19}}: \quad k_{19} \equiv \sum_{i} \sum_{j} f_{i j} x_{i}{ }^{2} y_{j}^{3}=\sum x_{i}{ }^{2} \sum y_{j}{ }^{4} b_{3}+\sum x_{i}{ }^{4} \sum y_{j}{ }^{4} b_{8}+\sum x_{i}{ }^{2} \sum y_{j}{ }^{6} b_{10} \\
& +\Sigma x_{i}{ }^{6} \Sigma y_{j}{ }^{4} b_{17}+\Sigma x_{i}{ }^{4} \Sigma y_{j}{ }^{6} b_{19}+2 x_{i}{ }^{2} \Sigma y_{j}{ }^{8} b_{21}
\end{aligned}
$$

$$
\begin{aligned}
\frac{\partial F}{\partial b_{20}}: & k_{20} \equiv \sum_{i} \sum_{j} f_{i j} x_{i} y_{j}^{4}=\Sigma x_{i}{ }^{2} \Sigma y_{j}{ }^{4} b_{2}+\Sigma x_{i}{ }^{4} \Sigma y_{j}{ }^{4} b_{7}+\Sigma x_{i}{ }^{2} \Sigma y_{j}{ }^{6} b_{9} \\
& +\sum x_{i}{ }^{6} \Sigma y_{j}{ }^{4} b_{16}+\Sigma x_{i}{ }^{4} \Sigma y_{j}{ }^{6} b_{18}+\Sigma x_{i}{ }^{2} \Sigma y_{j}{ }^{8} b_{20}
\end{aligned}
$$

$$
\begin{aligned}
& \frac{\partial F}{\partial b_{2 l}}: \quad k_{2 l} \equiv \sum_{i} \sum_{j} f_{i j} y_{j}^{5}=n \sum y_{j}{ }^{6} b_{3}+\sum x_{i}{ }^{2} \sum y_{j} b_{8}+n \dot{\Sigma} y_{j}{ }^{10} b_{10}+\sum x_{i}{ }^{4} \Sigma y_{j}{ }^{6} b_{17} \\
& +\sum x_{i}{ }^{2} \sum y_{j} b_{19}+n \sum y_{j}{ }^{10_{b_{21}}}
\end{aligned}
$$


From this set of equations we see that the variables are split into the following 'independent groups:

$$
\begin{aligned}
& b_{1}, b_{4}, b_{6}, b_{11}, b_{13}, b_{15} \text { - occur only in equations } 2,5,7,12,14 \text {, } \\
& b_{2}, b_{7}, b_{9}, b_{16}, b_{18}, b_{20} \text { - occur only in equations } 3,8,10,17,19 \text {, } \\
& \text { and } 21 \\
& b_{3}, b_{8}, b_{10}, b_{17}, b_{19}, b_{21} \text { - occur only in equations } 4,9,11,18,20 \text {, }
\end{aligned}
$$

Since we are interested only in central values and equations II.I.2 and II.l.7 show that these are functions of $b_{1}, b_{4}$, and $b_{6}$ only, we now consider only the six equations determining these values.

We again point out that - as in the one-dimensional case - all of the $k$ values are linear combinations of $f_{i j}$ and that our smoothing value $\left(b_{l}\right)$ and second derivative value $\left(b_{4}+b_{6}\right)$ will be some linear combination of the "k's". Hence to determine a weighting factor $w_{i j}$ at any point we need only use the contribution of that point to each of the summations involved in the " $k$ " values in the formulas. (Another way of saying this is that to determine the weight at each point we consider $f_{i j}$ to be a delta-function; $I$ at the point and 0 elsewhere.)

3. $3 \times 3$ Point Formulas. For $\mathrm{n} \times \mathrm{n}=3 \times 3: \mathrm{x}_{1}=\mathrm{y}_{1}=-1 ; \mathrm{x}_{2}=\mathrm{y}_{2}$ $=0 ; x_{3}=y_{3}=+1$ whence $\sum x_{i}^{2}=\sum y_{j}{ }^{2}=2$.

3.1. Linear. For a linear fit $\left(b_{4}=b_{5}=\ldots=b_{21}=0\right)$ the set of equations to be solved becomes.

$$
k_{1}=9 b_{1}
$$

Hence $f(0,0)=b_{1}=\frac{k_{1}}{9}$ and the smoothing matrix of weights is

$$
\frac{1}{9}\left[\begin{array}{lll}
1 & 1 & 1 \\
1 & 1 & 1 \\
1 & 1 & 1
\end{array}\right]
$$


3.2. Quadratic. For a quadratic fit $\left(b_{7}=b_{8}=\ldots=b_{21}=0\right)$ the set of equations to be solved becomes

$$
\begin{aligned}
& k_{1}=9 b_{1}+6 b_{4}+6 b_{6} \\
& k_{4}=6 b_{1}+6 b_{4}+4 b_{6} \\
& k_{6}=6 b_{1}+4 b_{4}+6 b_{6}
\end{aligned}
$$

The solution of this set of equations gives

$$
f(0,0)=b_{1}=\frac{1}{9}\left(5 k_{1}-3 k_{4}-3 k_{6}\right)
$$

and

$$
f^{\prime \prime}(0,0)_{a v}=b_{4}+b_{6}=\frac{1}{6}\left(3 k_{4}+3 k_{6}-4 k_{1}\right)
$$

Applying the definitions of $k_{1}, k_{4}$, and $k_{6}$ the matrix of weights for $f(0,0)$ is

$$
\frac{1}{9}\left[\begin{array}{rrr}
-1 & 2 & -1 \\
2 & 5 & 2 \\
-1 & 2 & -1
\end{array}\right]
$$

and for $f^{\prime \prime}(0,0)$ av the matrix is

$$
\frac{1}{6}\left[\begin{array}{rrr}
2 & -1 & 2 \\
-1 & -4 & -1 \\
2 & -1 & 2
\end{array}\right]
$$

4. $5 \times 5$ Point Formulas. For $\mathrm{n} \times \mathrm{n}=5 \times 5: \mathrm{x}_{1}=\mathrm{y}_{1}=-2 ; \mathrm{x}_{2}=\mathrm{y}_{2}$ $=-1 ; x_{3}=y_{3}=0 ; x_{4}=y_{4}=+1 ; x_{5}=y_{5}=+2$ whence $\Sigma x_{i}{ }^{2}=\Sigma y_{j}{ }^{2}=10$; $\Sigma \mathrm{x}_{i}{ }^{4}=\Sigma \mathrm{y}_{\mathrm{j}}{ }^{4}=34 ; \Sigma \mathrm{x}_{i}{ }^{6}=\Sigma \mathrm{y}_{\mathrm{j}}{ }^{6}=130 ; \Sigma \mathrm{x}_{i}{ }^{8}=\Sigma \mathrm{y}_{j}{ }^{8}=514$.

4.1. Linear. The linear fit gives $f(0,0)=b_{1}=\frac{k_{1}}{25}$, a uniform weighting for each element in the smoothing matrix. 
4.2. Quadratic or Cubic. For a quadratic or cubic fit $\left(b_{11}=b_{12}=\right.$ $\ldots=b_{21}=0$ ) the set of equations to be solved becomes

$$
\begin{aligned}
& \mathrm{k}_{1}=25 \mathrm{~b}_{1}+50 \mathrm{~b}_{4}+50 \mathrm{~b}_{6} \\
& \mathrm{k}_{4}=50 \mathrm{~b}_{1}+170 \mathrm{~b}_{4}+100 \mathrm{~b}_{6} \\
& \mathrm{k}_{6}=50 \mathrm{~b}_{1}+100 \mathrm{~b}_{4}+170 \mathrm{~b}_{6}
\end{aligned}
$$

The solution of this set of equations gives

$$
f(0,0)=b_{1}=\frac{1}{175}\left(27 k_{1}-5 k_{4}-5 k_{6}\right)
$$

and

$$
f^{\prime \prime}(0,0)_{a v}=b_{4}+b_{6}=\frac{1}{70}\left(-4 k_{1}+k_{4}+k_{6}\right)
$$

Applying the definitions of $k_{1}, k_{4}$, and $k_{6}$ the matrix of weights for $f(0,0)$ is

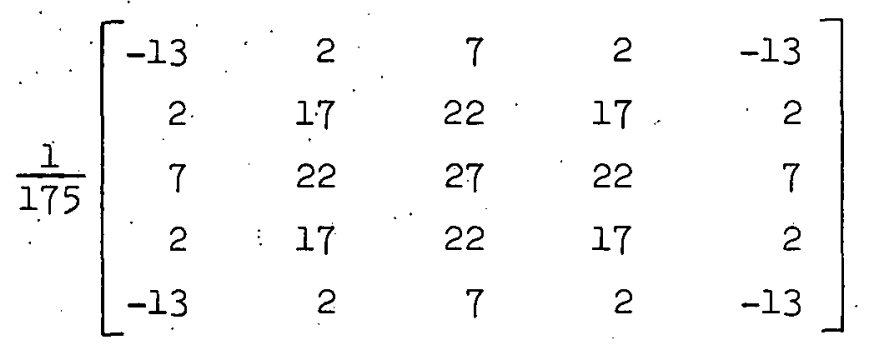

and for $f^{\prime \prime}(0,0)$ av the matrix is

$$
\frac{1}{70}\left[\begin{array}{rrrrr}
4 & 1 & 0 & 1 & 4 \\
1 & -2 & -3 & -2 & 1 \\
0 & -3 & -4 & -3 & 0 \\
1 & -2 & -3 & -2 & 1 \\
4 & 1 & 0 & 1 & 4
\end{array}\right]
$$


4.3. Quartic or Quintic. For a quartic or quintic fit the set of equations to be solved is

$$
\begin{aligned}
& k_{1}=25 b_{1}+50 b_{4}+50 b_{6}+170 b_{11}+100 b_{13}+170 b_{15} \\
& k_{4}=50 b_{1}+170 b_{4}+100 b_{6}+650 b_{11}+340 b_{13}+340 b_{15} \\
& k_{6}=50 b_{1}+100 b_{4}+170 b_{6}+340 b_{11}+340 b_{13}+650 b_{15} \\
& k_{11}=170 b_{1}+650 b_{4}+340 b_{6}+2570 b_{11}+1300 b_{13}+1156 b_{15} \\
& k_{13}=100 b_{1}+340 b_{4}+340 b_{6}+1300 b_{11}+1156 b_{13}+1300 b_{15} \\
& k_{15}=170 b_{1}+340 b_{4}+650 b_{6}+1156 b_{11}+1300 b_{13}+2570 b_{15}
\end{aligned}
$$

It is helpful to combine the above set of six equations into

$$
\begin{aligned}
& k_{1}=25 b_{1}+50\left(b_{4}+b_{6}\right)+100 b_{13}+170\left(b_{11}+b_{15}\right) \\
& \left(k_{4}+k_{6}\right)=100 b_{1}+270\left(b_{4}+b_{6}\right)+680 b_{13}+990\left(b_{11}+b_{15}\right)
\end{aligned}
$$$$
\left(k_{11}+k_{15}\right)=340 b_{1}+990\left(b_{4}+b_{6}\right)+2600 b_{13}+3726\left(b_{11}+b_{15}\right)
$$$$
k_{13}=100 b_{1}+340\left(b_{4}+b_{6}\right)+1156 b_{13}+1300\left(b_{11}+b_{15}\right)
$$

II. 4.3 .2

Then by solving the last of this group of equations for $b_{13}$ in terms of the other unknowns, substituting that quantity into the first three equations and simplifying we get:

$$
\begin{aligned}
& 289 k_{1}-25 k_{13}=(5)(945) b_{1}+(170)(35)\left(b_{4}+b_{6}\right)+(2)(8315)\left(b_{11}+b_{15}\right) \\
& 17\left(k_{4}+k_{6}\right)-10 k_{13}=(5)(140) b_{1}+(170)(7)\left(b_{4}+b_{6}\right)+(2)(1915)\left(b_{11}+b_{15}\right) \\
& 289\left(k_{11}+k_{15}\right)-650 k_{13}=(5)(6652) b_{1}+(170)(383)\left(b_{4}+b_{6}\right) \\
& +(2)(115907)\left(b_{11}+b_{15}\right)
\end{aligned}
$$


The solution of this set of equations gives

$$
\begin{aligned}
& f(0,0)=b_{1}=\frac{1}{4900}\left[2164 k_{1}-1425\left(k_{4}+k_{6}\right)+245\left(k_{11}+k_{15}\right)\right. \\
& \left.+100 k_{13}\right]
\end{aligned}
$$

and

$$
\begin{aligned}
& f^{\prime \prime}(0,0)_{a v}=b_{4}+b_{6}=\frac{1}{70560}\left[-41040 k_{1}+37523\left(k_{4}+k_{6}\right)\right. \\
& \left.-7595\left(k_{11}+k_{15}\right)-1440 k_{13}\right]
\end{aligned}
$$

Applying the definitions of $\mathrm{k}_{1}, \mathrm{k}_{4}, \mathrm{k}_{6}, \mathrm{k}_{11}, \mathrm{k}_{15}$, and $\mathrm{k}_{13}$ the matrix of weights for $f(0,0)$ is

$$
\frac{1}{1225}\left[\begin{array}{rrrrr}
51 & -99 & 96 & -99 & 51 \\
-99 & -24 & 246 & -24 & -99 \\
96 & 246 & 541 & 246 & 96 \\
-99 & -24 & 246 & -24 & -99 \\
51 & -99 & 96 & -99 & 51
\end{array}\right]
$$

and for $f^{\prime \prime}(0,0)$ the matrix is

$$
\frac{1}{17640}\left[\begin{array}{rrrrr}
-1734 & 2925 & -3117 & 2925 & -1734 \\
2925 & 4344 & -2778 & 4344 & 2925 \\
-3117 & -2778 & -10260 & -2778 & -3117 \\
2925 & 4344 & -2778 & 4344 & 2925 \\
-1734 & 2925 & -3117 & 2925 & -1734
\end{array}\right]
$$

5. $7 \times 7$ Point Formulas. For $\mathrm{n} \times \mathrm{n}=7 \times 7: \mathrm{x}_{1}=\mathrm{y}_{1}=-3 ; \mathrm{x}_{2}=\mathrm{y}_{2}$. $=-2 ; x_{3}=y_{3}=-1 ; x_{4}=y_{4}=0 ; x_{5}=y_{5}=1 ; x_{6}=y_{6}=2 ; x_{7}=y_{7}=3$ whence $\Sigma x_{i}^{2}=\Sigma y_{j}^{2}=28 ; \sum x_{i}^{4}=\Sigma y_{j}^{4}=196 ; \sum x_{i}{ }^{6}=\Sigma y_{j}{ }^{6}=1588 ; \sum x_{i}{ }^{8}=$ $\Sigma y_{j}^{8}=13636 ; \Sigma x_{i}^{2} \sum y_{j}^{2}=784 ; \sum x_{i}^{2} \sum y_{j}^{4}=\Sigma x_{i}^{4} \sum y_{j}^{2}=5488 ; \sum x_{i}^{2} \Sigma y_{j}^{6}=$ $\Sigma x_{i}^{6} \sum y_{j}^{2}=44464 ; \Sigma x_{i}^{4} \cdot \Sigma y_{j}^{4}=38416$. 
5.1. Linear. The linear fit gives $f(0,0)=b_{1}=\frac{k_{1}}{49}$, a uniform weighting for each element in the smoothing matrix.

5.2. Quadratic or Cubic. For a quadratic or cubic fit $\left(b_{11}=b_{12}=\right.$ $\ldots=b_{21}=0$ ) the set of equations to be solved becomes

$$
\begin{aligned}
& \mathrm{k}_{1}=49 \mathrm{~b}_{1}+196 \mathrm{~b}_{4}+196 \mathrm{~b}_{6} \\
& \mathrm{k}_{4}=196 \mathrm{~b}_{1}+(7)(196) \mathrm{b}_{4}+(4)(196) \mathrm{b}_{6} \\
& \mathrm{k}_{6}=196 \mathrm{~b}_{1}+(4)(196) \mathrm{b}_{4}+(7)(196) \mathrm{b}_{6}
\end{aligned}
$$

The solution of this set of equations gives

$$
f(0,0)=b_{1}=\frac{1}{147}\left(11 k_{1}-k_{4}-k_{6}\right)
$$

and

$$
f^{\prime \prime}(0,0)_{a v}=b_{4}+b_{6}=\frac{1}{588}\left(k_{4}+k_{6}-8 k_{1}\right)
$$

Applying the definitions of $\mathrm{k}_{1}, \mathrm{k}_{4}$, and $\mathrm{k}_{6}$ the matrix of weights for $f(0,0)$ is

$\frac{1}{47}\left[\begin{array}{rrrrrrr}-7 & -2 & 1 & 2 & 1 & -2 & -7 \\ -2 & 3 & 6 & 7 & 6 & 3 & -2 \\ 1 & 6 & 9 & 10 & 9 & 6 & 1 \\ 2 & 7 & 10 & 11 & 10 & 7 & 2 \\ 1 & 6 & 9 & 10 & 9 & 6 & 1 \\ -2 & 3 & 6 & 7 & 6 & 3 & 2 \\ -7 & -2 & 1 & 2 & 1 & -2 & 7\end{array}\right]$

II. 5.2 .4

and for $f^{\prime \prime}(0,0)$ av the matrix is

$$
\frac{1}{58 \overline{8}}\left[\begin{array}{rrrrrrr}
10 & 5 & 2 & 1 & 2 & 5 & 10 \\
5 & 0 & -3 & -4 & -3 & 0 & 5 \\
2 & -3 & -6 & -7 & -6 & -3 & 2 \\
1 & -4 & -7 & -8 & -7 & -4 & 1 \\
2 & -3 & -6 & -7 & -6 & -3 & 2 \\
5 & 0 & -3 & -4 & -3 & 0 & 5 \\
10 & 5 & 2 & 1 & 2 & 5 & 10
\end{array}\right]
$$


5.3. Quartic or Quintic. For a quartic or quintic fit the set of equations to be solved is

$$
\begin{aligned}
& \mathrm{k}_{1}=49 \mathrm{~b}_{1}+196 \mathrm{~b}_{4}+196 \mathrm{~b}_{6}+1372 \mathrm{~b}_{11}+784 \mathrm{~b}_{13}+1372 \mathrm{~b}_{15} \\
& \mathrm{k}_{4}=196 \mathrm{~b}_{1}+1372 \mathrm{~b}_{4}+784 \mathrm{~b}_{6}+11116 \mathrm{~b}_{11}+5488 \mathrm{~b}_{13}+5488 \mathrm{~b}_{15} \\
& \mathrm{k}_{6}=196 \mathrm{~b}_{1}+784 \mathrm{~b}_{4}+1372 \mathrm{~b}_{6}+5488 \mathrm{~b}_{11}+5488 \mathrm{~b}_{13}+11116 \mathrm{~b}_{15} \\
& \mathrm{k}_{11}=1372 \mathrm{~b}_{1}+11116 \mathrm{~b}_{4}+5488 \mathrm{~b}_{6}+95452 \mathrm{~b}_{11}+44464 \mathrm{~b}_{13}+38416 \mathrm{~b}_{15} \\
& \mathrm{k}_{13}=784 \mathrm{~b}_{1}+5488 \mathrm{~b}_{4}+5488 \mathrm{~b}_{6}+44464 \mathrm{~b}_{11}+38416 \mathrm{~b}_{13}+44464 \mathrm{~b}_{15} \\
& \mathrm{k}_{15}=1372 \mathrm{~b}_{1}+5488 \mathrm{~b}_{4}+11116 \mathrm{~b}_{6}+38416 \mathrm{~b}_{11}+44464 \mathrm{~b}_{13}+95452 \mathrm{~b}_{15}
\end{aligned}
$$

It is helpful to combine the above set of six equations into

$$
\begin{aligned}
& \frac{k_{1}}{49}=b_{1}+4\left(b_{4}+b_{6}\right)+16 b_{13}+28\left(b_{11}+b_{15}\right) \\
& \frac{\left(k_{4}+k_{6}\right)}{28}=14 b_{1}+77\left(b_{4}+b_{6}\right)+392 b_{13}+593\left(b_{11}+b_{15}\right) \\
& \frac{\left(k_{11}+k_{15}\right)}{28}=98 b_{1}+593\left(b_{4}+b_{6}\right)+3176 b_{13}+4781\left(b_{11}+b_{15}\right) \\
& \frac{k_{13}}{112}=7 b_{1}+49\left(b_{4}+b_{6}\right)+343 b_{13}+397\left(b_{11}+b_{15}\right)
\end{aligned}
$$

Then by solving the last of this group of equations for $b_{13}$ in terms of the other unknowns, substituting that quantity into the first three equations, and simplifying we get:

$$
\begin{aligned}
& 49 k_{1}-k_{13}=(147)(11) b_{1}+4116\left(b_{4}+b_{6}\right)+(84)(271)\left(b_{11}+b_{15}\right) \\
& 7\left(k_{4}+k_{6}\right)-2 k_{13}=(147)(8) b_{1}+4116\left(b_{4}+b_{6}\right)+(84)(325)\left(b_{11}+b_{15}\right) \\
& 343\left(k_{11}+k_{15}\right)-794 k_{13}=(147)(2168) b_{1}+(4116)(325)\left(b_{4}+b_{6}\right) \\
& +(84)(126337)\left(b_{11}+b_{15}\right)
\end{aligned}
$$


The solution of this set of equations gives

$$
\begin{aligned}
& f(0,0)=b_{1}=\frac{1}{19404}\left[3452 k_{1}-911\left(k_{4}+k_{6}\right)+63\left(k_{11}+k_{15}\right)\right. \\
& \left.+44 k_{13}\right]
\end{aligned}
$$

II. 5.3.4

and

$$
\begin{aligned}
& f_{\text {av }}^{\prime \prime}(0,0)=b_{4}+b_{6}=\frac{1}{(4)(38808)}\left[-14576 k_{1}+5457\left(k_{4}+k_{6}\right)\right. \\
& \left.-469\left(k_{11}+k_{15}\right)-176 k_{13}\right]
\end{aligned}
$$

Applying the definitions of $\mathrm{k}_{1}, \mathrm{k}_{4}, \mathrm{k}_{6}, \mathrm{k}_{11}, \mathrm{k}_{15}$, and $\mathrm{k}_{13}$ the matrix of weights for $f(0,0)$ is

$$
\frac{1}{4851}\left[\begin{array}{rrrrrrr}
206 & -174 & -24 & 89 & -24 & -174 & 206 \\
-174 & -279 & 36 & 204 & 36 & -279 & -174 \\
-24 & 36 & 450 & 651 & 450 & 36 & -24 \\
89 & 204 & 651 & 863 & 651 & 204 & 89 \\
-24 & 36 & 450 & 651 & 450 & 36 & -24 \\
-174 & -279 & 36 & 204 & 36 & -279 & -174 \\
206 & -174 & -24 & 89 & -24 & -174 & 206
\end{array}\right]
$$

and for $f^{\prime \prime}$ av $(0,0)$ the matrix of weights is

$$
\frac{1}{38808}\left[\begin{array}{rrrrrrr}
-1646 & 1134 & -12 & -863 & -12 & 1134 & -1644 \\
1134 & 2814 & 1008 & -63 & .1008 & 2814 & 1134 \\
-12 & 1008 & -1194 & -2397 & -1.191 & 1008 & -12 \\
-863 & -63 & -2397 & -3644 & -2397 & -63 & -863 \\
-12 & 1008 & -1194 & -2397 & -1194 & 1008 & -12 \\
1134 & 2814 & 1008 & -63 & 1008 & 2814 & 1134 \\
-1646 & 1134 & -12 & -863 & -12 & 1134 & -1646
\end{array}\right]
$$

II. 5.3 .7 
III. Example of Application*

'l'he $5 \times 5$ point quadratic smoothing matrix was applied to a nuclear medicine image with a low counting rate area to demonstrate the effectiveness of the least-squares processing when compared with a standard gaussian weighting method. The gaussian weight used is shown below. It is a "sharp" averager with one standard deviation per element spacing.

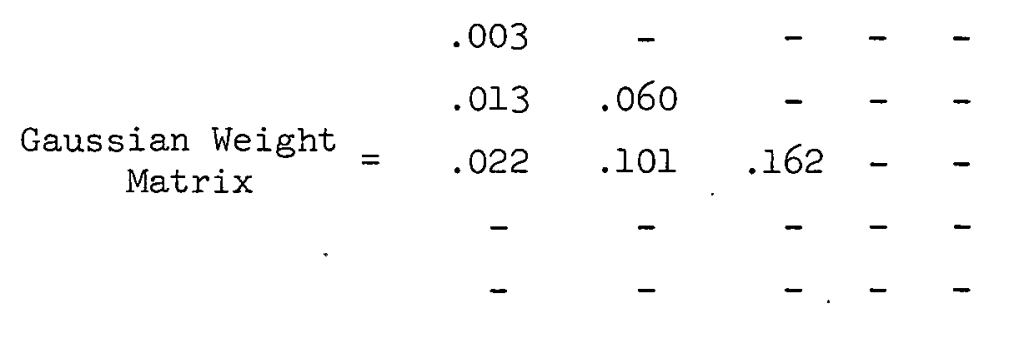

The image was an ordinary clinical scan with ${ }^{67} \mathrm{Ga}$ made on an Ohio Nuclear dual 8" rectilinear scanner. Only one $4 \mathrm{~K}$ section of this large scan is illustrated. The liver is at the upper edge of this section with the abdominal area in the lower part of the image.

Three processes were used in succession. The first pass was a bounding action in which the smoothing matrix was applied with weight 1 . The smoothed value was compared with this data point. If the point lay within the smoothed value \pm the square root of the smoothed value, the value was unchanged; if it lay outside the range, it was replaced by the smoothed value. The purpose is to remove bad points or statistically improbable values. Figure $I$ shows $Z$ cuts of the raw data and the least squares bounded image at the line marked in the image. After the bounding pass the images were smoothed once with weight 4 and once with weight 1 . Figure 2 shows the same line on the left with the simple gaussian weight and on the right with the least square quadratic fit. Note that the least squares process produces a smoother image while at the same time the ascending colon has

* Supplied by P. R. Bell, Medical Instrumentation Group. 
a greater relief from the region around it. The mean counting rate in this region is about 6 counts per picture element. Figure 3 shows a $\mathrm{Z}$ cut across the lower part of the liver. Note the steeper rise of the least squares lines and its rise to a higher value.

The time required for processing this image by the two methods was the same. 


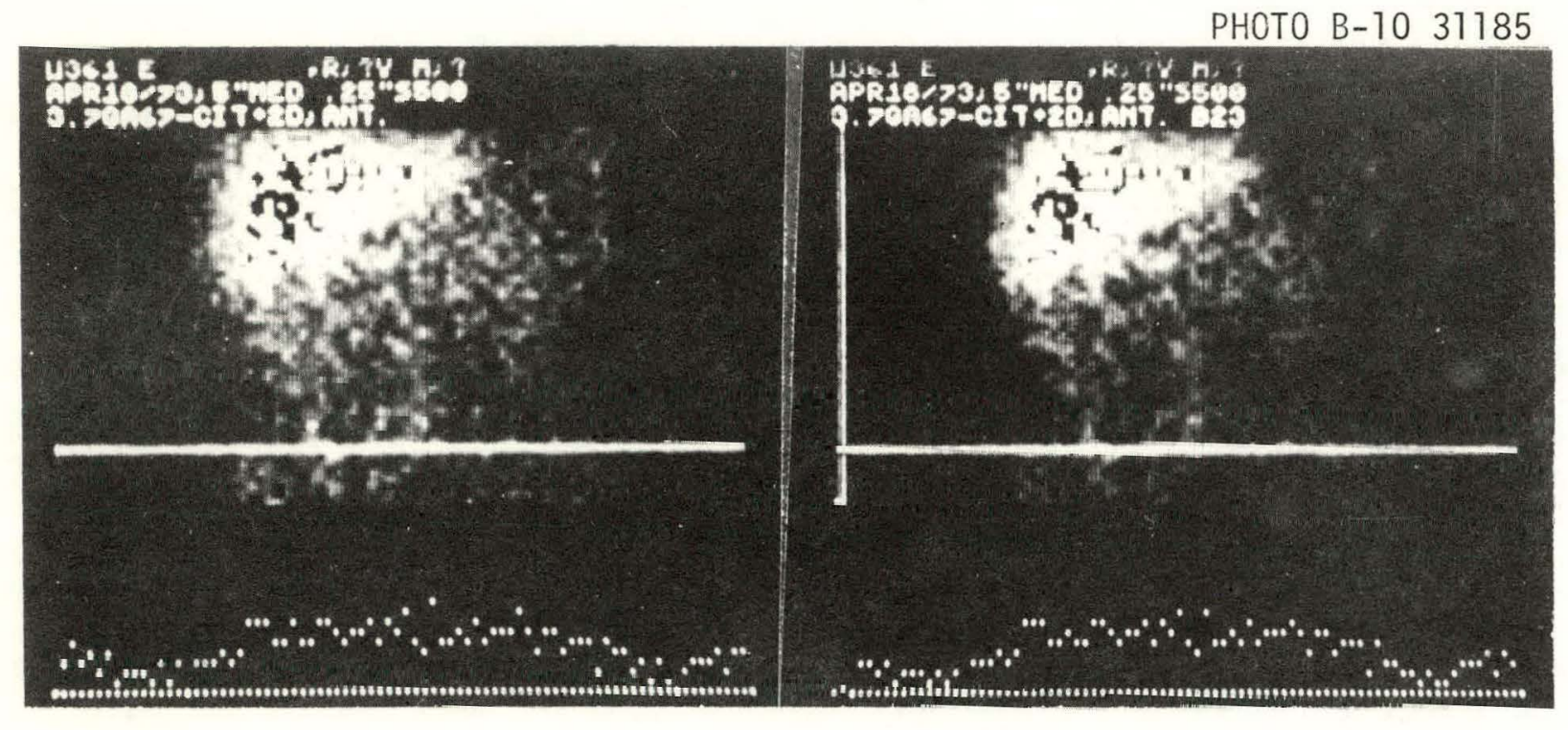

Fig. 1. Left is an unprocessed section of a ${ }^{67} \mathrm{Ga}$ image. The liver is at the top. The Z-cut crosses the ascending colon on the left central part of the image. The activity at the extreme left and right are the patient's arms. The count density near the ascending colon is about 6 counts/picture element.

The right image is that following bounding with the $5 \mathrm{x} 5$ point quadratic smoothing matrix. Note the reduction of the "sparkle" due to points with large statistical deviation in the image. 


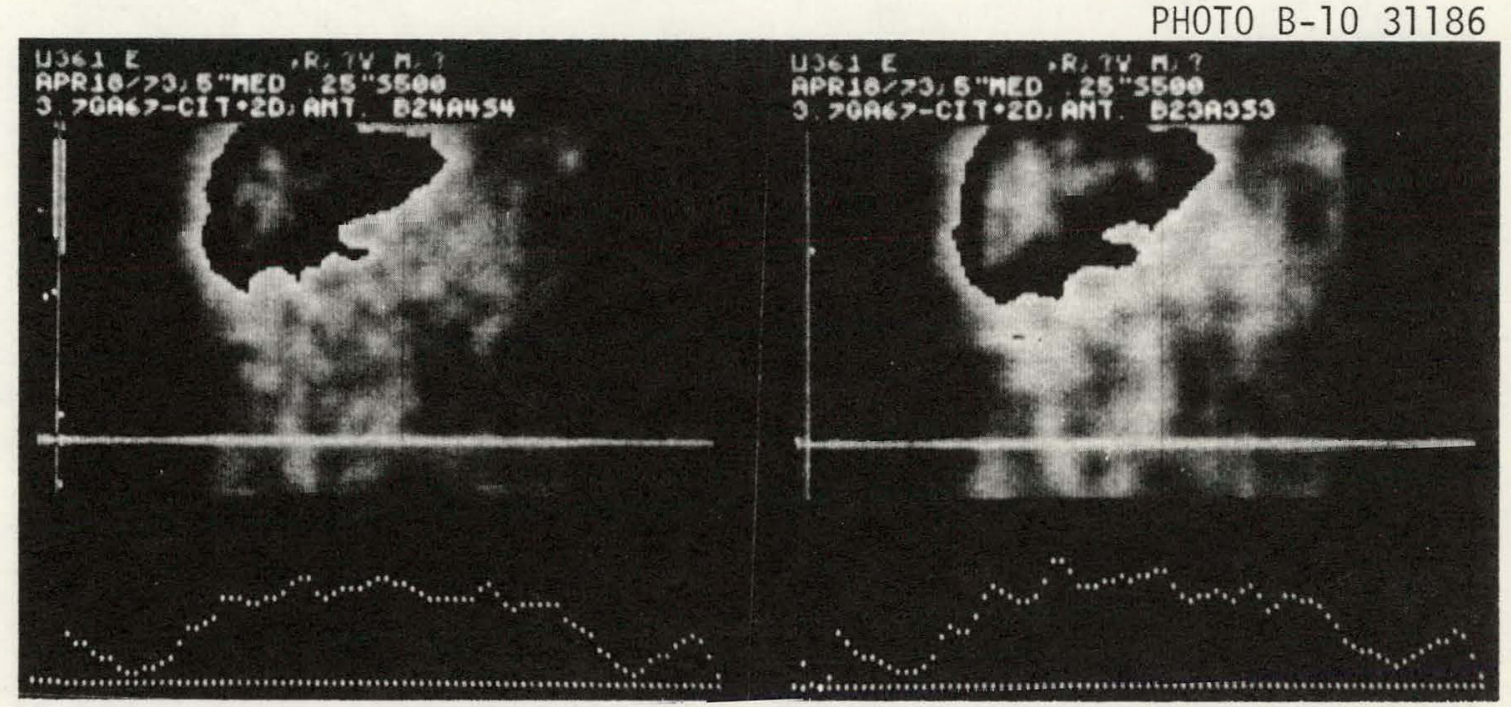

Fig. 2. Left image was produced by bounding followed by weight 4 smoothing and weight 1 smoothing all with the gaussian smoothing matrix. The right image was similarly processed using the $5 \times 5$ point least-squares quadratic smoothing matrix. Note the greater difference between the ascending colon and the region around it in the least-squares smoothed image. This is due to the greater suppression of this feature by the gaussian smoothing. Note the overall somewhat better smoothness of the least-squares processing. 


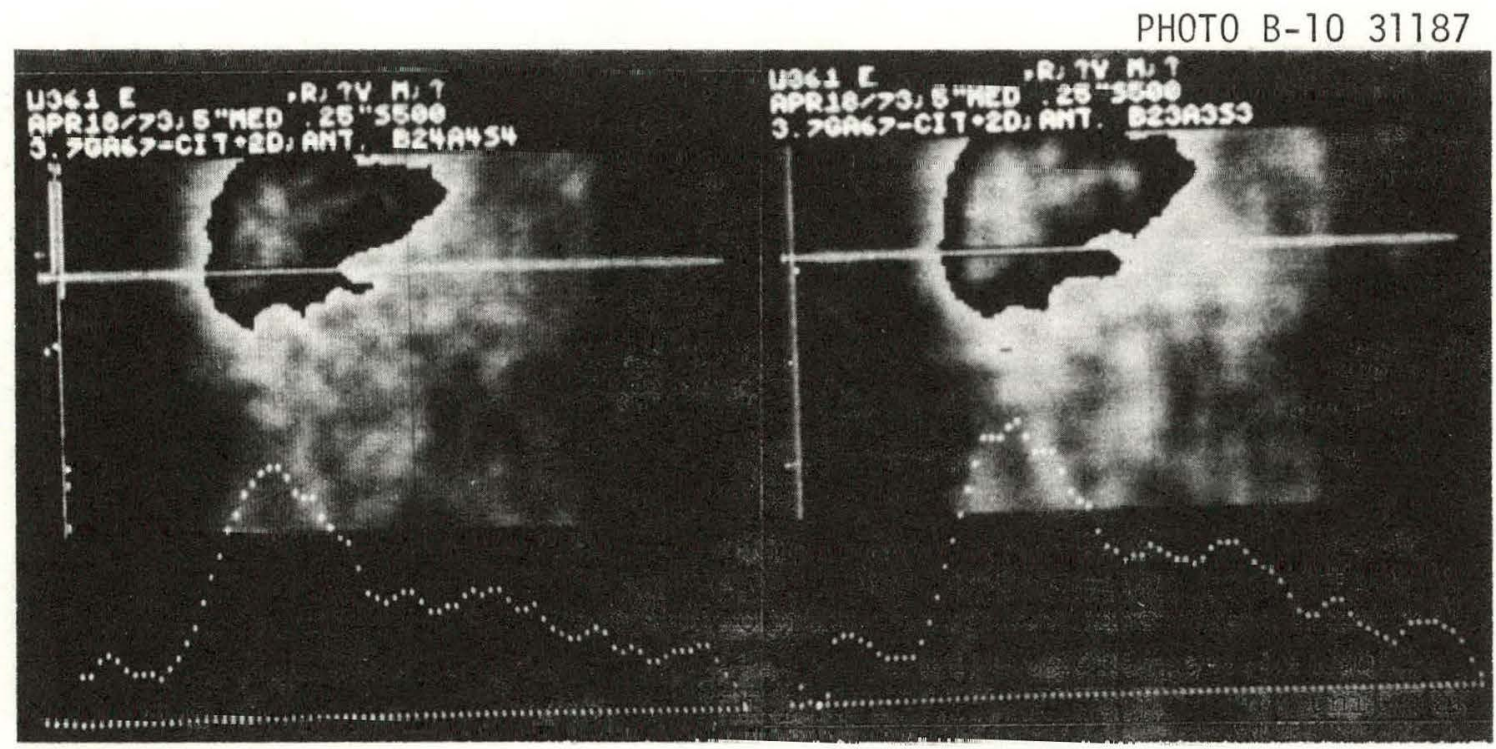

Fig. 3. Same images as in Fig. 2 with the Z-cut across the tip of the liver. Note the steeper and higher rise produced by the leastsquares processing. 
REFERENCES

1. Hildebrand, F. B., "Introduction to Numerical Analysis," McGraw-Hill Book Company, Inc., New York, 1956.

2. Savitzky, A. and Golay, J. E., Analytical Chemistry 36, 1627-39, July $(196 /)$.

3. Owen, G. E. and Premakoff, H., Physical Review, Vol. 74, No. 10, 1406-1412 (1948). 


\section{APPENDIX}

Once we have determined a least-squares fitting function (in either one or two dimensions) we can also give two other quantities of interest to image processing: the location and magnitude of the extreme (maximum or minimum) value of the function. We will give formulas for these quantities for quadratic fitting functions.

In the one dimensional case, $y=b_{1}+b_{2} x+b_{3} x^{2}$. so that the location of the extremum is given by setting $y^{\prime}=0$, i.e.

$$
\mathrm{x}_{\mathrm{EXT}}=\frac{-\mathrm{b}_{2}}{2 \mathrm{~b}_{3}}
$$

and the extreme value of the function is

$$
y_{E X T}=b_{1}-\frac{b_{2}^{2}}{4 b_{3}}
$$

If $y^{\prime \prime}$, i.e. $2 b_{3}$, is positive, the extremum is a minimum; if $y^{\prime \prime}$ is negative, the extremum is a maximum. The values of $b_{1}$ and $2 b_{3}$ are given by I.B.I.5 and I.B.1.6 for a 5 point fit and by I.B.2.5 and I.B.2.6 for a 7 point fit. From equation I.B. 6 we find $b_{2}=\sum_{i} f_{i} x_{i} / \sum_{i} x_{i}{ }^{2}$. Hence for the 5 point case

$$
b_{2}=\frac{1}{10}\left(-2 f_{-2}-f_{-1}+f_{1}+2 f_{2}\right)
$$

and for the 7 point case

$$
b_{2}=\frac{1}{28}\left(-3 f_{-3}-2 f_{-2}-f_{-1}+f_{1}+2 f_{2}+3 f_{3}\right)
$$

In the two dimensional case, $f(x, y)=b_{1}+b_{2} x+b_{3} y+b_{4} x^{2}+b_{5} x y$ $+b_{6} y^{2}$. The location of the extremum is given by the solution of the simultaneous equations

$$
\frac{\partial f}{\partial x}=b_{2}+2 b_{4} x+b_{3} y=0
$$

and

$$
\frac{\partial f}{\partial y}=b_{3}+b_{5} x+2 b_{6} y=0
$$


This solution is

$$
x_{\operatorname{EXT}}=\frac{-2 b_{2} b_{6}+b_{3} b_{5}}{4 b_{4} b_{6}-b_{5}^{2}}
$$

and

$$
Y_{E X T}=\frac{-2 b_{3} b_{4}+b_{2} b_{5}}{4 b_{4} b_{6}-b_{5}^{2}}
$$

The extreme value of the function is then given by

$$
f_{E X T}=b_{1}-\frac{\left(b_{2}{ }^{c_{2}} b_{6}-b_{2} b_{3} b_{5}+b_{3}{ }^{2} b_{4}\right)}{\left(4 b_{4} b_{6}-b_{5}^{2}\right)}
$$

Call the common quantity in the denominator of the above three expressions $\Delta$, i.e. $\Delta=4 \mathrm{~b}_{4} \mathrm{~b}_{6}-\mathrm{b}_{5}{ }^{2}$. The function will have a maximum value if $\Delta>0$ and either $b_{4}$ or $b_{6}<0$; the function will have a minimum value if $\Delta>0$ and either $b_{4}$ or $b_{6}>0$. If $\Delta<0$ (which is necessarily the case if $b_{4}$ and $b_{6}$ have opposite signs or are 0 ) the function has neither a maximum nor a minimum but rather a saddle point. Hence whenever there is the possibility of a maximum or a minimum $b_{4}$ and $b_{6}$ have the same sign.

For a $7 \times 7$ point fitting area for a quadratic, the value of the weighting matrix to be convolved with the experimental data to give $b_{1}$ is given by equation II.5.2.4. Equation II.5.2.3 gives $b_{4}+b_{6}=\frac{I}{588}\left(k_{4}+k_{6}\right.$ $-8 \mathrm{k}_{1}$ ) and it can be shown that

$$
\mathrm{b}_{4}=\frac{1}{588}\left(\mathrm{k}_{4}-4 \mathrm{k}_{1}\right)
$$

and

$$
\mathrm{b}_{6}=\frac{1}{588}\left(\mathrm{k}_{6}-4 \mathrm{k}_{1}\right)
$$

Equations II.2.3, II.2.4 and II.2.6 separately give the following solutions for $b_{2}, b_{3}$, and $b_{5}$. 


$$
\begin{aligned}
& b_{2}=\left(\sum_{i} \sum_{j} f_{i j} x_{i}\right) / n \sum_{i} x_{i}^{2} \\
& b_{3}=\left(\sum_{i} \sum_{j} f_{i j} y_{j}\right) / n \sum_{j} y_{j}^{2} \\
& b_{5}=\left(\sum_{i} \sum_{j} f_{i j} x_{i} y_{j}\right) / \sum_{i} x_{i}^{2} \sum_{j} y_{j}^{2}
\end{aligned}
$$

The weighting matrices to give these additional values are given below

W for $b_{2}=\frac{1}{196}\left[\begin{array}{ccccccc}-3 & -2 & -1 & 0 & 1 & 2 & 3 \\ -3 & -2 & -1 & 0 & 1 & 2 & 3 \\ -3 & -2 & -1 & 0 & 1 & 2 & 3 \\ -3 & -2 & -1 & 0 & 1 & 2 & 3 \\ -3 & -2 & -1 & 0 & 1 & 2 & 3 \\ -3 & -2 & -1 & 0 & 1 & 2 & 3 \\ -3 & -2 & -1 & 0 & 1 & 2 & 3\end{array}\right]$

W for $b_{3}=\frac{1}{196}\left[\begin{array}{rrrrrrr}3 & 3 & 3 & 3 & 3 & 3 & 3 \\ 2 & 2 & 2 & 2 & 2 & 2 & 2 \\ 1 & 1 & 1 & 1 & 1 & 1 & 1 \\ 0 & 0 & 0 & 0 & 0 & 0 & 0 \\ -1 & -1 & -1 & -1 & -1 & -1 & -1 \\ -2 & -2 & -2 & -2 & -2 & -2 & -2 \\ -3 & -3 & -3 & -3 & -3 & -3 & -3\end{array}\right]$

W for $b_{5}=\frac{1}{784}\left[\begin{array}{rrrrrrr}-9 & -6 & -3 & 0 & 3 & 6 & 9 \\ -6 & -4 & -2 & 0 & 2 & 4 & 6 \\ -3 & -2 & -1 & 0 & 1 & 2 & 3 \\ 0 & 0 & 0 & 0 & 0 & 0 & 0 \\ 3 & 2 & 1 & 0 & -1 & -2 & -3 \\ 6 & 4 & 2 & 0 & -2 & -4 & -6 \\ 9 & 6 & 3 & 0 & -3 & -6 & -9\end{array}\right]$ 
W for $b_{4}=\frac{1}{588}\left[\begin{array}{rrrrrrr}5 & 0 & -3 & -4 & -3 & 0 & 5 \\ 5 & 0 & -3 & -4 & -3 & 0 & 5 \\ 5 & 0 & -3 & -4 & -3 & 0 & 5 \\ 5 & 0 & -3 & -4 & -3 & 0 & 5 \\ 5 & 0 & -3 & -4 & -3 & 0 & 5 \\ 5 & 0 & -3 & -4 & -3 & 0 & 5 \\ 5 & 0 & -3 & -4 & -3 & 0 & 5\end{array}\right]$ 
THIS PAGE

\section{WAS INTENTIONALLY \\ LEFT BLANK}


ORNL-TM-5222

INTERNAL DISTRIBUTION

1. L. L. Anthony

2-2l. P. R. Bell

22. N. A. Betz

23. J.P. Breillatt

24. R. S. Dillon

25. J. E. Prancis, Jr.

26. R. M. French

27. R. A. Griesemer

28. R. L. Henne

29. W. J. McClain
30. C. D. Martin

31. C. R. Richmond

32. A. E. Stephens

33. J. B. Storer

34-35. Central Research Library

36. Document Reference Section

37-39. Laboratory Records

40. Laboratory Records, ORINL R.C.

41. ORNL Patent Office

EXTERNAL DISTRIBUTION

42. Dr. G. A. Andrews, Medical Division, Oak Ridge Associated Universities, Oak Ridge, Tennessee 37830

43. Dr. R. N. Beck, University of Chicago Medical Center, Argonne Cancer Research Hospital, 950 E. 59th Street, Chicago, Illinois 60637

44. Mr. R. T. Bell, Phelps-Dodge Western Exploration Office, Drawer 12l7, Douglas, Arizona. 85607

45. Dr. A. O. Bishop, Department of Electrical Engineering, University of Tennessee, Knoxville, Tennessee 37916

46. Dr. A. B. Brill, Department of Kadiology, Division of Nuclear Medicine, Vanderbilt University, Nashville, Tennessee

47. Dr. Valerie Brookeman, Department of Radiology, J. H. Miller Health Center, University of Florida, Gainesville, Florida 32610

48. Dr. Gordon Brownell, Massachusetts General Hospital, Physics Research Laboratory, Boston, Massachusetts 02114

49. Dr. Marshal Brucer, 5335 Via Celeste, Tucson, Arizona 85718 
50. Mr. Roger Cloutier, Chairman, Special Training, Oak Ridge Associated Universities, Oak Ridge, Tennessee 37830

51. Dr. Frank DeLand, Department of Radiation Medicine, Veterans Administration Hospital, Lexington, Kentucky 40406

52. Dr. Dale J. Fisher, Nuclear Medicine Service, Veterans Administration Hospital, Gainesville, Florida 32602

53. Dr. Mark Freeman, Nuclear Medicine Service, Veterans Administration Hosptial, Gainesville, Florida 32602

54. Mr. C. C. Harris, Duke University Medical Center, Box 3304, Durham, North Carolina 27710

55. Dr. Gerald Johnson, National Cancer Institute, Clinical Center, 1B51A Building \#10, National Institutes of Health, Bethesda, Maryland 20014

56. Dr. Ki-Hyon Kim, Physics Department, N. C. C. University, Durham, North Carolina 27707

57. Dr. D. E. Kuhl, Department of Radiology, Hospital of the University of Pennsylvania, 34th and Spruce, Philadelphia, Penrsylvania 19104

58. Dr. C. Lushbaugh, Chairman, Medical Division, Oak Ridge Associated Universities, Oak Ridge, Tennessee 37830

59. Dr. Felix J. Pircher, Chief, Nuclear Medical Service, Veterans Administration Hospital, 2002 Holcombe Boulevard, Houston, Texas 77031

60. Dr. James S. Robertson, Section of Diagnostic Nuclear Medicine, Department of Laboratory Medicine, Mayo Clinic, Rochester, Minnesota 55901

61. Dr. W. N. Tauxe, University of Alabama Hospital, Birmingham, Alabama

62. Dr. Charles David Teates, Assistant Professor of Radiology, University of Virginia, Charlottesville, Virginia 22901

63. Dr. H. N. Wagner, Johns-Hopkins Medical Institutions, Head, Divisions of Nuclear Medicine and Radiation Health, $615 \mathrm{~N}$. Wolfe Street, Baltimore, Maryland 21205

64. Dr. H. R. Wasson, Physical and Technical Programs, Energy Research and Development Administration, Washington, D. C. 20545

65. Dr. Robert H. Wilkinson, Assistant Professor of Radiology, Division of Nuclear Medicine, Duke University, Durham, North Carolina 27706 
66. Dr. R. W. Wood, Manager, Physical and Technical Programs, Energy Research and Development Administration, Washington, D. C. 20545

67. Research and Technical Support Division, Energy Research and Development Administration, Oak Ridge, Tennessee 37830

68-94. Technical Information Center (Abstracting Permitted), Oak Ridge, Tennessee 37830 